\title{
A Population-Based Study of Dystrophin Mutations in Canada
}

\author{
Jean K. Mah, Kathryn Selby, Craig Campbell, Amelie Nadeau, \\ Mark Tarnopolsky, Anna McCormick, Joseph M. Dooley, Hanna Kolski, \\ Andrew J. Skalsky, R. Garth Smith, David Buckley, Peter N. Ray, Grace Yoon, \\ on behalf of the Canadian Paediatric Neuromuscular Group
}

\begin{abstract}
Introduction: We carried out a population-based study of dystrophin mutations in patients followed by members of the Canadian Paediatric Neuromuscular Group (CPNG) over a ten-year period. Objectives: We aimed to describe the changes in diagnostic testing for dystrophinopathy and to determine the frequency of dystrophin mutations from 2000 to 2009. Methods: De-identified data containing the clinical phenotypes, diagnostic methods, and mutational reports from dystrophinopathy patients followed by CPNG centres from January 2000 to December 2009 were analyzed using descriptive statistics. Results: 773 patients had a confirmed diagnosis of dystrophinopathy based on genetic testing (97\%), muscle biopsy (2\%), or family history (1\%). 573 (74\%) had complete deletion/duplication analysis of all 79 exons or whole gene sequencing, resulting in 366 (64\%) deletions, 64 (11\%) duplications, and $143(25 \%)$ point mutations. The percentage of patients who were diagnosed using currently accepted genetic testing methods varied across Canada, with a mean of 63\% (SD 23). 246 (43\%) mutations involved exons 45 to 53 . The top ten deletions (n=147, 26\%) were exons 45-47, 45-48, 45, 45-50, 45-55, 51, 45-49, 45-52, 49-50, and 46-47. 169 (29\%) mutations involved exons 2 to 20. The most common duplications $(n=29,5.1 \%)$ were exons $2,2-7,2-17,3-7,8-11,10,10-11$, and 12. Conclusion: This is the most comprehensive report of dystrophin mutations in Canada. Consensus guidelines regarding the diagnostic approach to dystrophinopathy will hopefully reduce the geographical variation in mutation detection rates in the coming decade.
\end{abstract}

RÉSUMÉ: Étude de population sur les mutations de la dystrophine au Canada. Contexte : Nous avons effectué une étude de population sur les mutations du gène de la dystrophine chez des patients suivis par des membres du Canadian Paediatric Neuromuscular Group (CPNG) au cours d'une période de 10 ans. Objectifs : Notre but était de décrire l'évolution des tests diagnostiques des dystrophinopathies et de déterminer la fréquence des mutations du gène de la dystrophine de 2000 à 2009. Méthode : Nous avons analysé par des méthodes statistiques descriptives des données anomymisées, soit le phénotype clinique, les méthodes diagnostiques et l'identification de la mutation, chez des patients atteints de dystrophinopathies suivis dans des centres du CPNG de janvier 2000 à décembre 2009. Résultats : Un diagnostic de dystrophinopathie, confirmé par un test génétique (97\%), une biopsie musculaire (2\%) ou une histoire familiale (1\%), a été posé chez 773 patients. Chez 573 patients (74\%) une analyse complète des délétions/duplications des 79 exons ou un séquençage complet du gène a été effectué. Nous avons identifié des délétions chez 366 (64\%) patients, des duplications chez $64(11 \%)$ et des mutations ponctuelles chez 143 (25\%). Le pourcentage de patients chez qui le diagnostic de la maladie a été posé au moyen d'un test génétique dont la méthode de laboratoire est actuellement reconnue, était variable à travers le Canada, soit chez $63 \%$ des patients (ET 23). Deux cent quarante-six (43\%) des mutations étaient situées dans les exons 45 à 53. Les 10 délétions les plus fréquentes $(\mathrm{n}=147)$, soit $26 \%$, étaient situées dans les exons 45-47, 45-48, 45, 45-50, 45-55, 51, 45-49, 45-52, 49-50 et 46-47. Cent soixante-neuf mutations (29\%) étaient situées dans les exons 2 à 20. Les duplications les plus fréquentes $(\mathrm{n}=29)$, soit 5,1\% étaient situées dans les exons 2, 2-7, 2-17, 3-7, 8-11, 10, 10-11 et 12. Conclusion : Il s'agit du compte rendu le plus complet sur les mutations du gène de la dystrophine au Canada. D'ici une dizaine d'années, des lignes directrices de consensus concernant l'approche diagnostique des dystrophinopathies réduiront sans doute les disparités géographiques dans le taux de détection des mutations du gène de la dystrophine.

Can. J. Neurol. Sci. 2011; 38: 465-474

From the Division of Neurology (JKM), Department of Paediatrics, University of Calgary, Alberta Children's Hospital, Calgary; Division of Neurology (HK), Department of Paediatrics, University of Alberta, Stollery Children's Hospital, Edmonton, AB; Division of Neurology (KS), Department of Paediatrics, University of British Columbia, BC

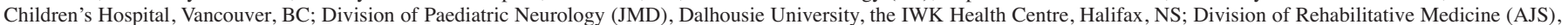
Department of Paediatrics, University of Manitoba, Winnipeg, MB; Department of Paediatrics (DB), Memorial University, Health Sciences Centre, St. John's, NL; Department of Neurology (AN), CHU Sainte-Justine, Montreal, QC; Division of Neuromuscular and Neurometabolic Disorders (MT), Department of Paediatrics and Medicine, McMaster Children's Hospital, McMaster University Medical Centre, Hamilton; Departments of Paediatrics (CC), Clinical Neurological Sciences and Epidemiology, University of Western

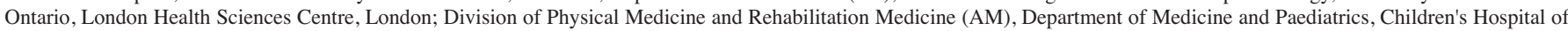

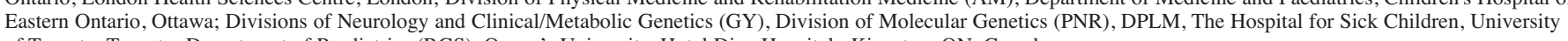
of Toronto, Toronto; Department of Paediatrics (RGS), Queen's University, Hotel Dieu Hospital, Kingston; ON, Canada.

Received September 30, 2010. Final Revisions Submitted November 19, 2010.

Correspondence to: Jean K. Mah, Alberta Children's Hospital, 2888 Shaganappi Trail NW, Calgary, Alberta, T3B 6A8, Canada. 
Duchenne and Becker muscular dystrophy (DBMD) are Xlinked recessive allelic disorders caused by mutations of the dystrophin gene on Xp21. Duchenne muscular dystrophy (OMIM \#310200) is the most common form of muscular dystrophy in childhood, with an incidence of 1 in 3,500 boys ${ }^{1}$. The absence or alteration of the dystrophin protein leads to calf pseudohypertrophy, muscle weakness, joint contractures, and cardiorespiratory dysfunction. Motor developmental delay, proximal weakness, and a variable degree of cognitive impairment are common presenting complaints. Duchenne muscular dystrophy (DMD) is associated with a severe phenotype, whereas Becker muscular dystrophy (BMD, OMIM \#300376) is a generally milder and more variable form of muscular dystrophy. Among individuals with DMD, progressive muscle necrosis leads to loss of independent ambulation by early adolescence, scoliosis, cardiomyopathy, respiratory insufficiency, and premature death as early as the third decade of life. The diagnosis of dystrophinopathy is usually considered after careful review of the clinical features, family history, laboratory evidence of markedly elevated serum creatine kinase (CK), and confirmed by investigations including muscle biopsy or molecular genetic testing.

The dystrophin gene contains 79 exons, which includes an actin-binding domain at the $\mathrm{N}$-terminus, 24 spectrin-like repeat units, a cysteine-rich dystroglycan binding site, and a C-terminal domain ${ }^{2,3}$. Two-thirds of dystrophin mutations are inherited; the remaining one-third occurs as de novo mutations ${ }^{4}$. The extremely large size of the dystrophin gene $(2.4 \mathrm{Mb})$ results in a complex mutational spectrum ( $>5000$ different reported mutations in the Leiden muscular dystrophy database, available via www.dmd.nl) as well as a high spontaneous mutation rate ${ }^{5}$. The primary objective of this study was to describe the changes in diagnostic testing for dystrophinopathy from 2000 to 2009 and to determine the frequency of dystrophin gene mutations among patients followed at participating Canadian Paediatric Neuromuscular Group (CPNG) centers during the same period.

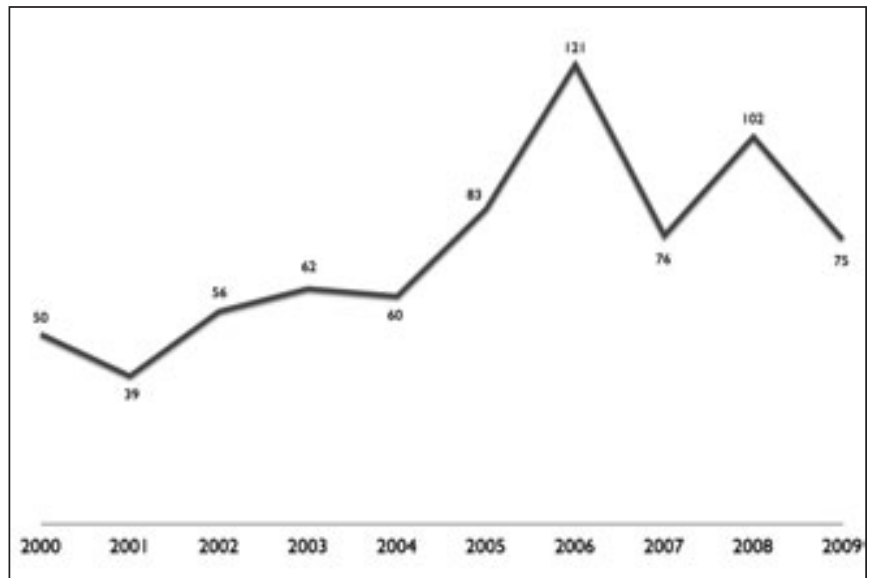

Figure 1: Total number of dystrophinopathy cases across Canada from 2000 to 2009.

\section{METHODS}

The CPNG includes neurologists, physiatrists, geneticists, and paediatricians across Canada who follow children with neuromuscular diseases in a tertiary care paediatric hospital or rehabilitative centre. The CPNG was established in 2005 as a collaborative effort to promote research and to enhance the clinical care of children with neuromuscular diseases. Informed consent was obtained for molecular genetic analysis, and the study was approved by the University of Calgary Conjoint Health Research and Ethics Board. De-identified data consisting of the clinical phenotypes, diagnostic methods, and molecular genetic reports from DBMD patients followed by participating CPNG centres from January 2000 to December 2009 were collected and verified at the Hospital for Sick Children in Toronto. The Molecular Genetics Laboratory at The Hospital for Sick Children is the only accredited laboratory in Canada for dystrophin DNA sequencing. Results were summarized using descriptive statistics and analyzed using Stata version 9.0 (StataCorp, College Station, TX) statistical software. Categorical variables were expressed as frequencies and percentages. Continuous variables were reported as means with standard deviations (SD) or as medians with interquartile ranges if the data were skewed. Bivariate comparisons were made using Pearson's chi-square or Fisher's exact tests for categorical variables and unpaired Student's t-tests or one-way analyses of variance for continuous variables. All tests were two-tailed, and $\mathrm{p}$ values less than 0.05 were considered to be statistically significant.

\section{RESUlts}

\section{a. Phenotypic presentation}

There were 773 individuals with dystrophinopathy, including $529(68 \%)$ with DMD and $137(18 \%)$ with BMD phenotype.

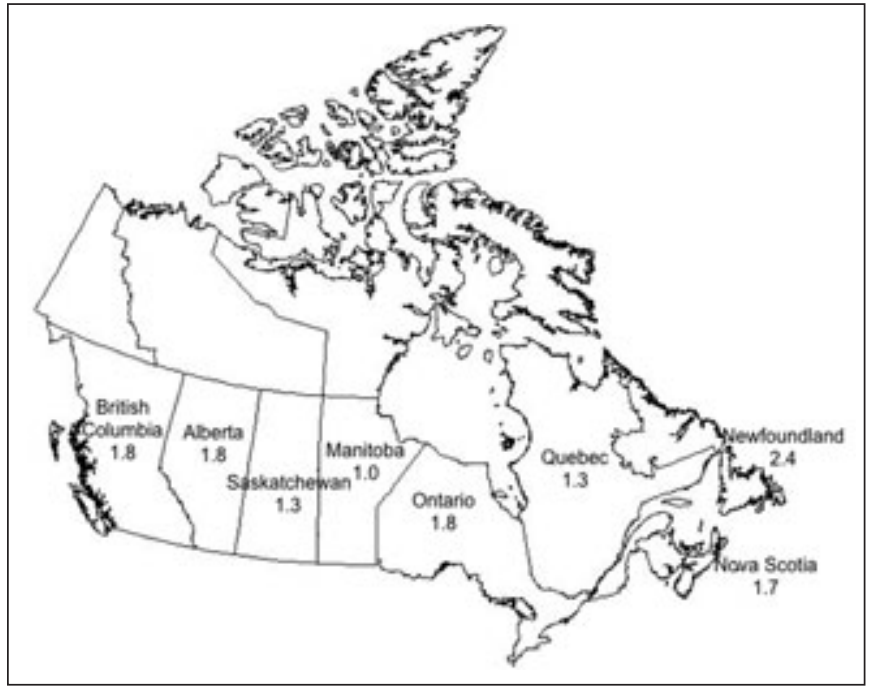

Figure 2: Estimated prevalence of dystrophinopathy per 10,000 males across Canada. 
One hundred and seven (14\%) had an unspecified phenotype due to young age at time of diagnosis, lack of follow-up, and/or insufficient clinical details to allow differentiation between DMD and BMD. Most (97\%) of the 773 patients had a confirmed diagnosis of dystrophinopathy based on molecular genetic testing, including multiplex ligation-dependent probe amplification (MLPA) in 439 (57\%) cases, multiplex polymerase chain reaction (PCR) in $168(22 \%)$ cases, and sequencing of the entire dystrophin gene in $142(18 \%)$. The remaining 24 cases were confirmed based on muscle biopsy $(2 \%)$ or positive family history in the setting of typical clinical features for DMD or BMD (1\%) and a markedly elevated serum CK.

\section{b. Distribution of cases and estimated prevalence}

The average number of DBMD cases was 72 per year (SD: 25) (see Figure 1). There was a gradual increase in the number of reported DBMD cases over time, with a significant difference between year 2000-2004 (mean 53, SD 9) and year 2005-2009 (mean 91, SD 20, unpaired Student's T-test $\mathrm{p}=0.009$ ). Using the Canadian 2006 census data of $31,612,897$ people (including $15,475,970$ males and $16,136,927$ females) as the mean population for the ten year period from 2000 to 2009, the estimated prevalence of dystrophinopathy across Canada was $773 / 5,000,555$, or 1.5 per 10,000 males between the ages of 0 to 24 years $(95 \%$ confidence interval, CI, 1.2 - 1.9). The estimated prevalence for each province is summarized in Figure 2. There was no significant difference in the prevalence of dystrophinopathy among the provinces.

\section{c. Common mutation sites}

Five hundred and seventy three (74\%) subjects including 377 DMD, 105 BMD, and 91 with unspecified phenotype had complete analysis of all 79 exons or whole gene sequencing, resulting in 366 (64\%) deletions, 64 (11\%) duplications, and 143 $(25 \%)$ point mutations. Seven out of the ten most common deletions from this study were identical to those reported by White et al from the Leiden DMD mutation database ${ }^{6}$; in contrast, only three out of the top ten duplications were shared by the Leiden database (see Supplementary Table 1).

Among the 366 individuals with confirmed deletions involving one or more exons, a major mutational hotspot around exons 45 to 55 was found. The ten most common deletions (147 out of 366, approximately $40 \%$ ) reported in this study were exons $45-47(n=27,7 \%), 45-48(n=17,5 \%), 45(n=15,4 \%)$, $45-50(n=15,4 \%), 45-55(n=14,4 \%), 51(n=12,3 \%), 45-49$ $(\mathrm{n}=12,3 \%), 45-52(\mathrm{n}=12,3 \%), 49-50(\mathrm{n}=12,3 \%)$, and $46-$ $47(n=11,3 \%)$ (see Figure 3).

Among the 64 individuals with confirmed duplications involving one or more exons, a minor mutational hotspot around exons 2 to 20 was noted (see Figure 4). The most common duplications (29 out of 64, approximately $45 \%$ ) were exons 2 $(n=13,20 \%), 2-7(n=2,3 \%), 2-17(n=2,3 \%), 3-7(n=2,3 \%)$, $8-11(\mathrm{n}=2,3 \%), 10(\mathrm{n}=2,3 \%), 10-11(\mathrm{n}=2,3 \%), 12(\mathrm{n}=2$, $3 \%), 63-69(n=2,3 \%)$, and $64-67(n=2,3 \%)$. The reason for the increased mutageneic susceptibility around the major (exons 45 - 55) and minor (exons 2 - 20) hotspot regions remains unclear.

\begin{tabular}{|c|c|c|c|c|}
\hline RANK & $\begin{array}{c}\text { Deletion } \\
\text { (current study) }\end{array}$ & $\begin{array}{c}\text { Deletion } \\
\text { (White et al) }\end{array}$ & $\begin{array}{c}\text { Duplication } \\
\text { (current study) }\end{array}$ & $\begin{array}{l}\text { Duplication } \\
\text { (White et al) }\end{array}$ \\
\hline 1 & $45-47$ & $45-47$ & 2 & 2 \\
\hline 2 & $45-48$ & 45 & $2-7$ & $2-7$ \\
\hline 3 & 45 & $48-50$ & $2-17$ & 8-9 \\
\hline 4 & $45-50$ & $45-48$ & $3-7$ & $3-7$ \\
\hline 5 & $45-55$ & $45-50$ & $8-11$ & 51 \\
\hline 6 & 51 & 51 & 10 & $3-4$ \\
\hline 7 & $45-49$ & 44 & $10-11$ & 44 \\
\hline 8 & $45-52$ & $49-50$ & 12 & $8-13$ \\
\hline 9 & $49-50$ & $3-7$ & 63-69 & 2-11 \\
\hline 10 & $46-47$ & $45-52$ & $64-67$ & $3-11$ \\
\hline
\end{tabular}

*Common mutations are highlighted in bold 


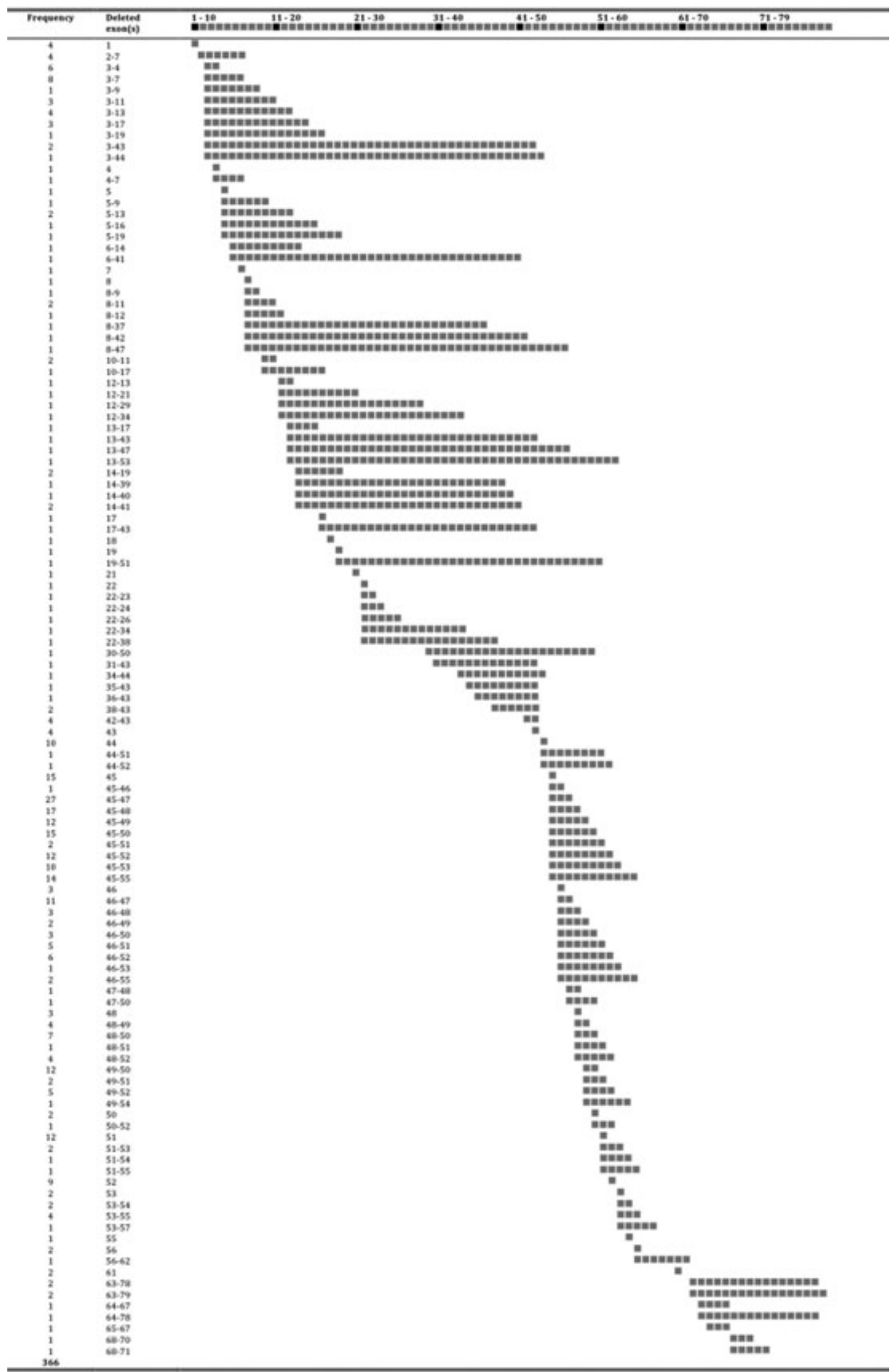

Figure 3: Confirmed deletions of the dystrophin gene among CPNG centers 
Among the 143 cases with point mutations, 67 (47\%) had nonsense mutations, 43 (30\%) had point deletions, and $33(23 \%)$ had small substitutions, duplications and insertions. In contrast to deletions or duplications involving one or more exons, point mutations were detected throughout the dystrophin gene, with no specific regions prone to increased mutation rates (see Supplementary Table 2).

\section{d. Correlation with clinical phenotypes}

We examined cases with confirmed boundaries of deletions or duplications to determine if the changes resulted in either an outof-frame or in-frame mutation. According to the reading frame rule $^{5}$, an out-of-frame mutation is associated with a DMD phenotype, with inability to walk unsupported by 12 to 14 yearsof-age and/or evidence of absent dystrophin on muscle biopsy. An in-frame mutation is associated with a milder BMD phenotype, with ability to walk unassisted beyond 16 years-ofage and/or reduced dystrophin by immunohistochemistry. We found that a number of identical deletions or duplications involving one or more exons were reportedly associated with both a severe DMD as well as a milder BMD phenotype. These deletions and effects of the mutations using the Leiden DMD gene reading frame checker (www.dmd.nl/index.html) were: exons 3 - 7, an out-of-frame deletion (4 DMD, 4 BMD); 3 - 11, an out-of-frame deletion (1 DMD, 1 BMD); 45 - 47, an in-frame deletion (5 DMD, 21 BMD); 45 - 49, an in-frame deletion (5 DMD, 6 BMD); 45 - 51, an in-frame deletion (1 DMD, 1 BMD); 45 - 53, an in-frame deletion (1 DMD, 6 BMD); and 45 - 55, an in-frame deletion (1 DMD, 12 BMD) (see Supplementary Table 3 ). The only duplication reportedly associated with $10 \mathrm{DMD}$ as well as 1 BMD phenotype was exon 2, an out-of-frame mutation (see Supplementary Table 4). In total, at least 13 DMD cases had an in-frame deletion, whereas six BMD cases possessed an outof-frame deletion. The variable phenotypes may be related in part to known exceptions to the reading frame rule, which has been reported in up $10 \%$ of dystrophin gene mutations with either deletions or duplications ${ }^{5}$.

The majority of the 67 cases with nonsense mutations were reportedly associated with a DMD phenotype, except for a premature stop codon mutation in exon 38 (c.5404C $>\mathrm{T}$ $(\mathrm{Q} 1802 \mathrm{X})$ ) in two subjects that was associated with a milder BMD phenotype (see Supplementary Table 2). Only one nonsense mutation involving exon 60 (c.8944C>T (p.R2982X)) was reportedly shared by two brothers.

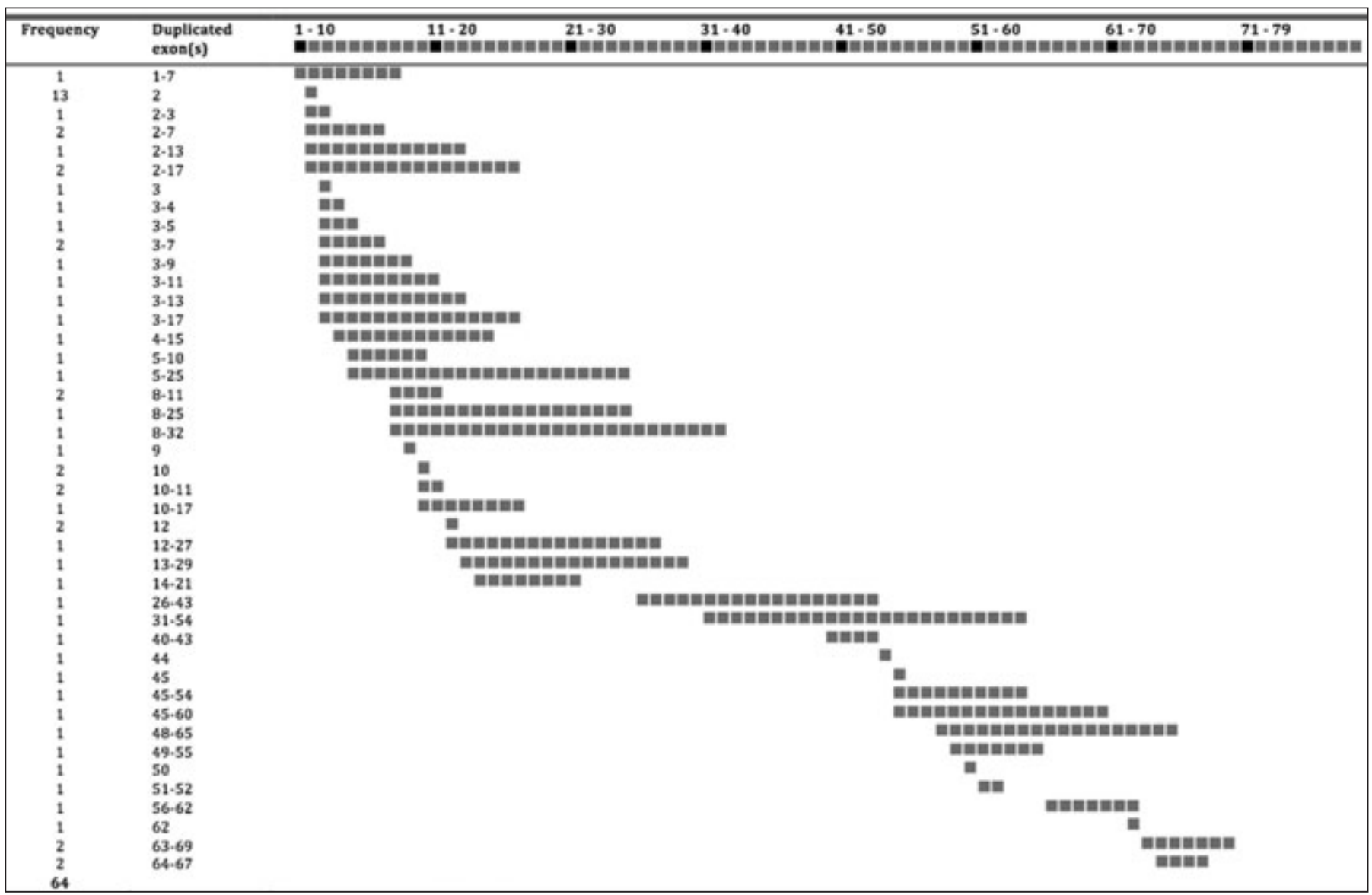

Figure 4: Confirmed duplications of the dystrophin gene among CPNG centers 
Supplementary Table 2: Confirmed point mutations among Canadian sites*

\begin{tabular}{|c|c|c|c|c|c|c|c|}
\hline Exon & Type & Report & Phenotype & Exon & Type & Report & Phenotype \\
\hline 2 & Pt-del & c.53delA (p.Lys18Argfs) & BMD & 20 & Pt-stop & c.2440G $>\mathrm{T}$ (p.Glu814X) & $\overline{\mathrm{MD}}$ \\
\hline 6 & Pt-del & c.367_368delGT & MD & 21 & Pt-stop & c. $2665 \mathrm{C}>\mathrm{T}$ (p.Arg889X) & DMD \\
\hline 8 & Pt-del & c.676_678delAAG (p.K226del) & BMD & 21 & Pt-stop & c. $2677 \mathrm{C}>\mathrm{T}$ (p.Gln $893 \mathrm{X})$ & DMD \\
\hline 10 & Pt-del & c. $113 \overline{4}$ delG & DMD & 23 & Pt-stop & c. $3151 \mathrm{C}>\mathrm{T}(\mathrm{p} . \mathrm{Arg} 1051 \mathrm{X})$ & DMD \\
\hline 12 & Pt-del & c. 1390 delC & DMD & 24 & Pt-stop & c. $3256 \mathrm{~A}>\mathrm{T}(\mathrm{p} . \mathrm{K} 1086 \mathrm{X})$ & DMD \\
\hline 14 & Pt-del & c.1666delG & MD & 25 & Pt-stop & c. $3414 \mathrm{G}>\mathrm{A}(\mathrm{p} . \operatorname{Trp} 1138 \mathrm{X})$ & DMD \\
\hline 15 & Pt-del & c.1784_1788del (p.Glu595fs) & DMD & 27 & Pt-stop & c. $3625 \mathrm{C}>\mathrm{T}(\mathrm{p} . \mathrm{G} \ln 1209 \mathrm{X})$ & DMD \\
\hline 15 & Pt-del & c.1784_1788delAAATG (p.Glu595fs) & MD & 32 & Pt-stop & c. $4375 \mathrm{C}>\mathrm{T}(\mathrm{p} . \mathrm{R} 1459 \mathrm{X})$ & DMD \\
\hline 16 & Pt-del & c.1943delC & DMD & 32 & Pt-stop & c. $4483 \mathrm{C}>\mathrm{T}(\mathrm{p} . \mathrm{Gln} 1495 \mathrm{X})$ & DMD \\
\hline 18 & Pt-del & c.2183_2184delTA (p.Ile728fs) & DMD & 33 & Pt-stop & c. $4666 \mathrm{G}>\mathrm{T}(\mathrm{p} . \mathrm{Gly} 1556 \mathrm{X})$ & DMD \\
\hline 18 & Pt-del & c.2183_2184delTA (p.Ile728fs) & DMD & 34 & Pt-stop & c. $4729 \mathrm{C}>\mathrm{T}(\mathrm{p} . \operatorname{Arg} 1577 \mathrm{X})$ & DMD \\
\hline 18 & Pt-del & c.2281_2285del (p.Glu761fs) & MD & 34 & Pt-stop & c. $4814 \mathrm{G}>\mathrm{T}(\mathrm{p} . \mathrm{Q} 1536 \mathrm{X})$ & DMD \\
\hline 18 & Pt-del & c.2281_2285del (p.Glu761fs) & DMD & 36 & Pt-stop & c. $5131 \mathrm{C}>\mathrm{T}(\mathrm{p} . \mathrm{G} \ln 1711 \mathrm{X})$ & DMD \\
\hline 20 & Pt-del & c.2384delG & MD & 36 & Pt-stop & c. $5131 \mathrm{C}>\mathrm{T}(\mathrm{p} . \mathrm{Gln} 1711 \mathrm{X})$ & DMD \\
\hline 21 & Pt-del & c.2636_2654del (p.Arg879fs) & DMD & 37 & Pt-stop & c. $5159 \mathrm{~T}>\mathrm{G}(\mathrm{p}$. Leu 1720X) & DMD \\
\hline 24 & Pt-del & c.3179delT (p.Leu1060fs) & DMD & 38 & Pt-stop & c. $5404 \mathrm{C}>\mathrm{T}(\mathrm{p} . \mathrm{Q} 1802 \mathrm{X})$ & BMD \\
\hline 26 & Pt-del & c.3347_3350delAGAA & DMD & 38 & Pt-stop & c. $5404 \mathrm{C}>\mathrm{T}(\mathrm{p} . \mathrm{Q} 1802 \mathrm{X})$ & BMD \\
\hline 27 & Pt-del & c.3737-3743del (p.Asn1246fs) & DMD & 39 & Pt-stop & c. $5521 \mathrm{G}>\mathrm{T}$ (p.Glu1841X) & BMD \\
\hline 29 & Pt-del & c.3963ㄹelT (p.Asn1321fs) & MD & 39 & Pt-stop & c. $5495 \mathrm{C}->\mathrm{T}(\mathrm{p} . \mathrm{R} 1763 \mathrm{X})$ & MD \\
\hline 30 & Pt-del & c.4156delC (p.Leu1386fs) & DMD & 41 & Pt-stop & c.5878G $>\mathrm{T}$ (p.Glu1960X) & MD \\
\hline 35 & Pt-del & c.4876_4887del (p.Val1625_Lys1629del) & BMD & 41 & Pt-stop & c.5878G $>\mathrm{T}$ (p.Glu1960X) & DMD \\
\hline 35 & Pt-del & c.4876_4887del (p.Val1625_Lys1629del) & BMD & 41 & Pt-stop & c. $5878 \mathrm{G}>\mathrm{T}$ (p.Glu1960X) & DMD \\
\hline 36 & Pt-del & c.5118_5119delGA (p.Lys1708GlufsX9) & DMD & 42 & Pt-stop & c. $5938 \mathrm{G}>\mathrm{T}$ (p.Glu1980X) & DMD \\
\hline 38 & Pt-del & c.5401_5402delAT & DMD & 43 & Pt-stop & c. $6250 \mathrm{C}>\mathrm{T}(\mathrm{p} . \mathrm{Gln} 2084 \mathrm{X})$ & MD \\
\hline 38 & Pt-del & 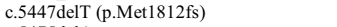 & MD & 45 & Pt-stop & c. $6460 \mathrm{C}>\mathrm{T}(\mathrm{p} . \mathrm{Gln} 2154 \mathrm{X})$ & DMD \\
\hline 39 & Pt-del & c.5475delA & DMD & 47 & Pt-stop & c. $6905 \mathrm{G}>\mathrm{A}($ (p.Trp2303X) & DMD \\
\hline 47 & Pt-del & c.6804_6807del & DMD & 52 & Pt-stop & c. $7561 \mathrm{G}>\mathrm{T}(\mathrm{p} . \mathrm{E} 2521 \mathrm{X})$ & DMD \\
\hline 47 & Pt-del & c. 6809 del $4 \mathrm{bp}$ & DMD & 54 & Pt-stop & c. $8009 \mathrm{G}>\mathrm{A}(\mathrm{p} . \mathrm{W} 2670 \mathrm{X})$ & DMD \\
\hline 50 & Pt-del & c.7292_7296del & DMD & 55 & Pt-stop & c. $8161 \mathrm{~A}>\mathrm{T}(\mathrm{p} . \mathrm{Lys} 2721 \mathrm{X})$ & DMD \\
\hline 55 & Pt-del & c. $8167 \overline{\mathrm{de}} \mathrm{AA}$ & DMD & 55 & Pt-stop & c. $8214 \mathrm{G}>\mathrm{A}($ p.W2738X) & DMD \\
\hline 57 & Pt-del & c. 8542 delC (p.His $2848 \mathrm{fs}$ ) & DMD & 57 & Pt-stop & c. $8464 C>T(p . G \ln 2822 X)$ & DMD \\
\hline 58 & Pt-del & c.8605_8606del (p.Val2869fs) & DMD & 57 & Pt-stop & c. $8483 \mathrm{~T}>\mathrm{G}(\mathrm{p} . \mathrm{L} 2828 \mathrm{X})$ & DMD \\
\hline 59 & Pt-del & c.8912_8913del (p.Leu2971fs) & DMD & 60 & Pt-stop & c. $8944 \mathrm{C}>\mathrm{T}(\mathrm{p} . \mathrm{R} 2982 \mathrm{X})$ & DMD \\
\hline 65 & Pt-del & c. $9556 \mathrm{del} ; 9562 \_9563+13 \mathrm{del}$ & DMD & 60 & Pt-stop & c. $8944 \mathrm{C}>\mathrm{T}(\mathrm{p} . \mathrm{R} 2982 \mathrm{X})$ & DMD \\
\hline 66 & Pt-del & c.9596_9597delTT & MD & 60 & Pt-stop & c. $9001 \mathrm{C}>\mathrm{T}(\mathrm{p} . \mathrm{G} \ln 3002 \mathrm{X})$ & DMD \\
\hline 68 & Pt-del & c.9854_9863del (p.Leu3329X) & DMD & 61 & Pt-stop & c. $9100 \mathrm{C}>\mathrm{T}(\mathrm{p} . \mathrm{Arg} 3034 \mathrm{X})$ & DMD \\
\hline 70 & Pt-del & c.10101_10103del (p.Glu3367del) & DMD & 61 & Pt-stop & c. $9100 \mathrm{C}>\mathrm{T}(\mathrm{p} . \mathrm{Arg} 3034 \mathrm{X})$ & DMD \\
\hline 74 & Pt-del & c.10441- delC (p.Gln3481fs) & DMD & 61 & Pt-stop & c. $9100 \mathrm{C}>\mathrm{T}$ (p.Arg3034X) & DMD \\
\hline 74 & Pt-del & c.10454delT (p.Leu3485Argfs) & DMD & 63 & Pt-stop & c. $9276 \mathrm{C}>\mathrm{A}($ p. $\mathrm{Y} 3092 \mathrm{X})$ & DMD \\
\hline 74 & Pt-del & c.10454delT (p.Leu3485fs) & MD & 67 & Pt-stop & c. $9661 \mathrm{C}>\mathrm{T}(\mathrm{p} . \mathrm{G} \ln 3221 \mathrm{X})$ & MD \\
\hline 74 & Pt-del & c.10454delT (p.Leu3485fs) & MD & 68 & Pt-stop & c. $9851 \mathrm{G}>\mathrm{A}(\mathrm{p} . \mathrm{W} 3284 \mathrm{X})$ & DMD \\
\hline 74 & Pt-del & c.10454delT (p.Leu3485fs) & DMD & 70 & Pt-stop & c. $10108 \mathrm{C}>\mathrm{T}(\mathrm{p} . \mathrm{Arg} 3370 \mathrm{X})$ & DMD \\
\hline 75 & Pt-del & c.10744delC (p.His3582fs) & DMD & 70 & Pt-stop & c. $10108 \mathrm{C}>\mathrm{T}(\mathrm{R} 3370 \mathrm{X})$ & DMD \\
\hline 26 & Pt-delins & c.3494_3497delATCTinsCCTTCGTGC & DMD & 70 & Pt-stop & c. $10171 \mathrm{C}>\mathrm{T}(\mathrm{R} 3391 \mathrm{X})$ & DMD \\
\hline 66 & Pt-delins & c.9564-1_9564delinsTT [r.(spl?)] & DMD & 70 & Pt-stop & c. $10171 \mathrm{C}>\mathrm{T}(\mathrm{R} 3391 \mathrm{X})$ & DMD \\
\hline 17 & Pt-dup & c.2044dupA & DMD & 5 & Pt-sub & c. $265-1 \mathrm{G}>\mathrm{C}$ & DMD \\
\hline 27 & Pt-dup & c.3713dupA (p.Glu1239fs) & MD & 5 & Pt-sub & c. $265-2 \mathrm{~A}>\mathrm{G}$ & DMD \\
\hline 63 & Pt-dup & c.9260_9278dup & DMD & 5 & Pt-sub & c. $357+1 \mathrm{G}>\mathrm{T}[\mathrm{r} .(\mathrm{spl})]$ & MD \\
\hline 27 & Pt-inser & c.3705_3706ins 11 & MD & 6 & Pt-sub & c. $511 \mathrm{G}>\mathrm{C}$ (p.Ala171Pro) & BMD \\
\hline 62 & Pt-inser & c.9221_9222insAT & DMD & 7 & Pt-sub & c. $649+1 \mathrm{G}>\mathrm{T}$ & DMD \\
\hline 43 & Pt-inser & c.6290_6291ins80bp & DMD & 7 & Pt-sub & c. $649+1 \mathrm{G}>\mathrm{T}$ & DMD \\
\hline 5 & Pt-stop & c.355C $>\mathrm{T}(\mathrm{p} . \mathrm{Q} 119 \mathrm{X})$ & DMD & 7 & Pt-sub & c. $531-1 \mathrm{G}>\mathrm{C}$ & DMD \\
\hline 6 & Pt-stop & c. $433 \mathrm{C}>\mathrm{T}$ (p.R145X) & DMD & 7 & Pt-sub & c. $649+2 \mathrm{~T}>\mathrm{C}$ & DMD \\
\hline 8 & Pt-stop & c.783dupT (p.Lys262X) & DMD & 9 & Pt-sub & c. $961-1 \mathrm{G}>\mathrm{A}[\mathrm{r} .(\mathrm{spl} ?)]$ & DMD \\
\hline 8 & Pt-stop & c. $829 \mathrm{C}>\mathrm{T}(\mathrm{p} . \mathrm{G} \ln 277 \mathrm{X})$ & DMD & 13 & Pt-sub & c. $1483-2 \mathrm{~A}>\mathrm{C}$ & BMD \\
\hline 8 & Pt-stop & c. 799C $>$ T (p.Q267X) & DMD & 13 & Pt-sub & c. $1483-1 \mathrm{G}>\mathrm{C}$ & BMD \\
\hline 11 & Pt-stop & c.1292G>A (p.Trp431X) & DMD & 16 & Pt-sub & c. $1992+2 \mathrm{~T}>\mathrm{G}$ & DMD \\
\hline 11 & Pt-stop & c. $1207 \mathrm{G}>\mathrm{T}$ (p.Gly403X) & DMD & 18 & Pt-sub & c. $2292+2 \mathrm{~T}>\mathrm{C}$ & DMD \\
\hline 12 & Pt-stop & c. $1357 \mathrm{C}>\mathrm{T}(\mathrm{p} . \mathrm{G} \ln 453 \mathrm{X})$ & DMD & 26 & Pt-sub & c. $3603+3 \mathrm{~A}>\mathrm{T}$ & BMD \\
\hline 14 & Pt-stop & c. $1615 \mathrm{C}>\mathrm{T}$ (p.Arg539X) & DMD & 26 & Pt-sub & c. $3603+3 \mathrm{~A}>\mathrm{T}$ & BMD \\
\hline 14 & Pt-stop & c. $1637 \mathrm{G}>\mathrm{A}(\mathrm{p} . \operatorname{Trp} 546 \mathrm{X})$ & MD & 48 & Pt-sub & c. $7096 \mathrm{~A}>\mathrm{C}(\mathrm{p} . \mathrm{K} 2366 \mathrm{Q})$ & DMD \\
\hline 14 & Pt-stop & c. $1637 \mathrm{G}>$ A (p.Trp546X) & DMD & 52 & Pt-sub & c. $7571 \mathrm{G}>\mathrm{A}$ (p.Arg2524His) & MD \\
\hline 14 & Pt-stop & c. $1702 \mathrm{C}>\mathrm{T}(\mathrm{p} . \mathrm{Q} 568 \mathrm{X})$ & DMD & 56 & Pt-sub & c. $8218-2 A>G$ & DMD \\
\hline 16 & Pt-stop & c. $1990 \mathrm{C}>\mathrm{T}(\mathrm{p} . \mathrm{G} \ln 664 \mathrm{X})$ & DMD & 56 & Pt-sub & c. $8218-2 \mathrm{~A}>\mathrm{G}$ & DMD \\
\hline 18 & Pt-stop & c.2276_2292+70delins40bp & DMD & 59 & Pt-sub & c. $8729 \mathrm{~A}>\mathrm{T}$ (p.Glu2910Val); c.8734A $>\mathrm{G}$ (p.Asn2912Asp) & DMD \\
\hline 18 & Pt-stop & c. $2276 \_2292+70$ delins $40 \mathrm{bp}$ & DMD & 64 & Pt-sub & c. $9361+1 \mathrm{G}>\mathrm{A}$ & DMD \\
\hline 19 & Pt-stop & c.2302C $>\mathrm{T}(\mathrm{p} . \mathrm{R} 798 \mathrm{X})$ & DMD & 68 & Pt-sub & c. $9974+3 \mathrm{~A}>\mathrm{T}$ & BMD \\
\hline 19 & Pt-stop & c. $2302 \mathrm{C}>\mathrm{T}(\mathrm{p} . \operatorname{Arg} 768 \mathrm{X})$ & DMD & 68 & Pt-sub & c. $9937 \mathrm{~T}>\mathrm{G}(\mathrm{p} . \mathrm{C} 3313 \mathrm{G})$ & MD \\
\hline 19 & Pt-stop & c. $2353 \mathrm{C}>\mathrm{T}(\mathrm{p} . \mathrm{G} \ln 785 \mathrm{X})$ & DMD & 69 & Pt-sub & c. $9975-2 \mathrm{~A}>\mathrm{G}$ & DMD \\
\hline 19 & Pt-stop & c. $2332 \mathrm{C}>\mathrm{T}(\mathrm{p} . \mathrm{G} \ln 778 \mathrm{X})$ & DMD & 69 & Pt-sub & c. $9975-2 \mathrm{~A}>\mathrm{G}$ & MD \\
\hline 19 & Pt-stop & c. $2332 \mathrm{C}>\mathrm{T}(\mathrm{p} . \mathrm{G} \ln 778 \mathrm{X})$ & DMD & & & & \\
\hline
\end{tabular}

* Identical mutations shared by two or more individuals are highlighted in grey; DMD refers to Duchenne muscular dystrophy, BMD refers to Becker muscular dystrophy, and MD denotes an unspecifed phenotype.

\section{e. Change in diagnostic approaches over time}

Only $13(2 \%)$ of all dystrophinopathy cases were diagnosed based on muscle biopsy alone, and they were performed at or before the year 2000. The muscle biopsy criteria for the diagnosis of DBMD was based on the pathological features of a dystrophic process with diffuse variation in fiber size, necrosis, and fibrosis, plus either absent (DMD) or reduced (BMD) dystrophin by immunohistochemistry. As seen in Figure 5, there was a noticeable change in the molecular diagnostic methods over time. Multiplex polymerase chain reactions (PCR) of a subset of exons was the most common diagnostic test initially (up to $98 \%$ of cases) in 2000 , but it was gradually replaced by MLPA and other molecular genetic tests that interrogated all 79 exons. Overall, MLPA confirmed the mutations in 431 (75\%) cases. Complete gene sequencing was introduced in 2002, and it was used in 142 cases (25\%) between 2002 to 2009 if MLPA failed to detect a deletion or duplication in the dystrophin gene. 
Supplementary Table 3: Confirmed deletions among CPNG sites*

\begin{tabular}{|c|c|c|c|c|c|}
\hline Exons & Frequency & Phenotypes & Exons & Frequency & Phenotypes \\
\hline 1 & 4 & $\mathrm{DMD}(2), \mathrm{MD}(2)$ & & 4 & \\
\hline $2-7$ & 4 & BMD & $\begin{array}{l}43 \\
44\end{array}$ & $\begin{array}{l}4 \\
10\end{array}$ & $\begin{array}{l}\text { DMD } \\
\text { DMD (9), MD (1) }\end{array}$ \\
\hline $3-4$ & 6 & BMD (5), MD (1) & 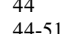 & 10 & DMD (9), MD (1) \\
\hline $3-7$ & 8 & DMD (4), BMD (4) & $\begin{array}{l}44-51 \\
44-52\end{array}$ & 1 & $\begin{array}{l}\text { DMD } \\
\text { DMD }\end{array}$ \\
\hline 3-9 & 1 & MD & $44-52$ & & \\
\hline $3-11$ & 3 & DMD (1), BMD (1), MD (1) & & 15 & DMD \\
\hline $3-13$ & 4 & DMD (3), MD (1) & $\begin{array}{l}45-46 \\
45-47\end{array}$ & $\begin{array}{l}1 \\
27\end{array}$ & $\begin{array}{l}\text { DMD } \\
\text { BMD (21). DMD (5). MD (1) }\end{array}$ \\
\hline $3-17$ & 3 & DMD & $\begin{array}{r}45-4 / \\
45-48\end{array}$ & 17 & $\begin{array}{l}\text { BMD (21), DMD (5), MD (1) } \\
\text { BMD (15), MD (2) }\end{array}$ \\
\hline $3-19$ & 1 & DMD & $\begin{array}{l}45-48 \\
45-49\end{array}$ & 12 & $\begin{array}{l}\mathrm{BMD}(15), \mathrm{MD}(2) \\
\mathrm{BMD}(6) \text { DMD (5) MD (1) }\end{array}$ \\
\hline $3-43$ & 2 & $\mathrm{MD}$ & $\begin{array}{l}45-49 \\
45-50\end{array}$ & $\begin{array}{l}12 \\
15\end{array}$ & $\begin{array}{l}\text { BMD (6), DMD (5), MD (1) } \\
\operatorname{DMD}(11), M D \\
\text { (4) }\end{array}$ \\
\hline $3-44$ & 1 & DMD & $\begin{array}{l}45500 \\
45-51\end{array}$ & 2 & BMD (1), DMD (1) \\
\hline $4-7$ & 1 & BMD & $45-52$ & & \\
\hline $\begin{array}{l}4-1 \\
5\end{array}$ & 1 & $\begin{array}{l}\text { BMD } \\
\text { BMD }\end{array}$ & $45-53$ & 10 & BMD (6), DMD (1), MD (3) \\
\hline $5-9$ & 1 & $\begin{array}{l}\text { BMD } \\
\text { MD }\end{array}$ & $45-55$ & 14 & $\operatorname{BMD}(12), \operatorname{DMD}(1), \mathrm{MD}(1)$ \\
\hline $5-13$ & 2 & MD & 46 & 3 & DMD \\
\hline $5-16$ & 1 & $\begin{array}{l}\text { DMD } \\
\text { BMD }\end{array}$ & $46-47$ & 11 & DMD (9), MD (2) \\
\hline $5-19$ & 1 & $\begin{array}{l}\text { BMD } \\
\text { DMD }\end{array}$ & $46-48$ & 3 & $\mathrm{DMD}(2), \mathrm{MD}(1)$ \\
\hline $6-14$ & 1 & $\begin{array}{l}\text { DMD } \\
\text { DMD }\end{array}$ & $46-49$ & 2 & DMD \\
\hline $6-41$ & 1 & $\begin{array}{l}\text { DMD } \\
\text { DMD }\end{array}$ & $46-50$ & 3 & $\mathrm{DMD}(1), \mathrm{MD}(2)$ \\
\hline 7 & 1 & $\begin{array}{l}\text { DMD } \\
\text { DMD }\end{array}$ & $46-51$ & 5 & DMD (4), MD (1) \\
\hline 8 & 1 & $\begin{array}{l}\text { DMD } \\
\text { DMD }\end{array}$ & $46-52$ & 6 & DMD (5), MD (1) \\
\hline $8-9$ & 1 & $\begin{array}{l}\text { DMD } \\
\text { DMD }\end{array}$ & $46-53$ & 1 & DMD \\
\hline $8-11$ & 2 & $\begin{array}{l}\text { DMD } \\
\text { DMD }\end{array}$ & $46-55$ & 2 & DMD \\
\hline $8-12$ & 1 & $\begin{array}{l}\text { DMD } \\
\text { DMD }\end{array}$ & $47-48$ & 1 & BMD \\
\hline $8-37$ & 1 & $\begin{array}{l}\text { DMD } \\
\text { MD }\end{array}$ & $47-50$ & 1 & DMD \\
\hline $8-42$ & 1 & $\begin{array}{l}\text { MD } \\
\text { DMD }\end{array}$ & 48 & 3 & DMD \\
\hline $8-47$ & 1 & $\begin{array}{l}\text { DMD } \\
\text { MD }\end{array}$ & $48-49$ & 4 & BMD (2), MD (2) \\
\hline $10-11$ & 2 & $\begin{array}{l}\text { MD } \\
\text { DMD }\end{array}$ & $48-50$ & 7 & $\operatorname{DMD}(2), \mathrm{MD}(5)$ \\
\hline $10-17$ & 1 & $\begin{array}{l}\text { DMD } \\
\text { DMD }\end{array}$ & $48-51$ & 1 & BMD \\
\hline $12-13$ & 1 & $\begin{array}{l}\text { DMD } \\
\text { DMD }\end{array}$ & $48-52$ & 4 & DMD \\
\hline $12-21$ & 1 & $\begin{array}{l}\text { DMD } \\
\text { DMD }\end{array}$ & $49-50$ & 12 & DMD (10), MD (2) \\
\hline $12-29$ & 1 & $\begin{array}{l}\text { DMD } \\
\text { DMD }\end{array}$ & $49-51$ & 2 & DMD (1), MD (1) \\
\hline $12-34$ & 1 & DMD & $49-52$ & 5 & DMD (3), MD (2) \\
\hline $13-17$ & 1 & DMD & $49-54$ & 1 & DMD \\
\hline $13-43$ & 1 & $\begin{array}{l}\text { DMD } \\
\text { DMD }\end{array}$ & 50 & 2 & DMD \\
\hline $13-47$ & 1 & $\begin{array}{l}\text { DMD } \\
\text { DMD }\end{array}$ & $50-52$ & 1 & DMD \\
\hline $13-53$ & 1 & $\begin{array}{l}\text { DMD } \\
\text { MD }\end{array}$ & 51 & 12 & DMD (10), MD (2) \\
\hline $14-19$ & 2 & $\begin{array}{l}\mathrm{MD} \\
\mathrm{DMD}(1), \mathrm{MD}(1)\end{array}$ & $51-53$ & 2 & DMD (1), MD (1) \\
\hline $14-39$ & 1 & $\begin{array}{l}\text { DMD (1), MD (1) } \\
\text { BMD }\end{array}$ & $51-54$ & 1 & DMD \\
\hline $14-40$ & 1 & $\begin{array}{l}\text { BMD } \\
\text { BMD }\end{array}$ & $51-55$ & 1 & DMD \\
\hline $14-41$ & 2 & $\begin{array}{l}\text { BMD } \\
\text { BMD (1), MD (1) }\end{array}$ & 52 & 9 & DMD \\
\hline 17 & 1 & $\begin{array}{l}\text { BMD (1), MD (1) } \\
\text { DMD }\end{array}$ & 53 & 2 & DMD \\
\hline $17-43$ & 1 & $\begin{array}{l}\text { DMD } \\
\text { DMD }\end{array}$ & $53-54$ & 2 & DMD \\
\hline 18 & 1 & $\begin{array}{l}\text { DMD } \\
\text { DMD }\end{array}$ & $53-55$ & 4 & DMD \\
\hline 19 & 1 & $\begin{array}{l}\text { DMD } \\
\text { MD }\end{array}$ & $53-57$ & 1 & DMD \\
\hline $19-51$ & 1 & $\begin{array}{l}\text { MD } \\
\text { DMD }\end{array}$ & 55 & 1 & DMD \\
\hline 21 & 1 & $\begin{array}{l}\text { DMD } \\
\text { DMD }\end{array}$ & 56 & 2 & DMD \\
\hline 22 & 1 & $\begin{array}{l}\text { DMD } \\
\text { DMD }\end{array}$ & $56-62$ & 1 & DMD \\
\hline $22-23$ & 1 & $\begin{array}{l}\text { DMD } \\
\text { DMD }\end{array}$ & 61 & 2 & DMD \\
\hline $22-24$ & 1 & $\begin{array}{l}\text { DMD } \\
\text { MD }\end{array}$ & $63-78$ & 2 & DMD \\
\hline $22-26$ & 1 & DMD & $63-79$ & 2 & DMD \\
\hline $22-34$ & 1 & $\begin{array}{l}\text { DMD } \\
\text { DMD }\end{array}$ & $64-67$ & 1 & MD \\
\hline $22-38$ & 1 & $\begin{array}{l}\text { DMD } \\
\text { DMD }\end{array}$ & $64-78$ & 1 & DMD \\
\hline $30-50$ & 1 & $\begin{array}{l}\text { DMD } \\
\text { MD }\end{array}$ & $65-67$ & 1 & DMD \\
\hline $31-43$ & 1 & $\begin{array}{l}\text { MD } \\
\text { DMD }\end{array}$ & $68-70$ & 1 & MD \\
\hline $34-44$ & 1 & $\begin{array}{l}\text { DMD } \\
\text { DMD }\end{array}$ & $68-71$ & 1 & DMD \\
\hline $35-43$ & 1 & $\begin{array}{l}\text { DMD } \\
\text { DMD }\end{array}$ & Total & 366 & \\
\hline $36-43$ & 1 & DMD & & & \\
\hline $38-43$ & 2 & DMD & & & \\
\hline $42-43$ & 4 & DMD (3), MD (1) & & & \\
\hline
\end{tabular}

* Identical mutations with both Duchenne (DMD) and Becker (BMD) muscular dystrophy phenotypes are highlighted in grey; MD denotes an unspecifed phenotype.

\section{f. Variability in access to complete genetic testing across the provinces}

Patients were considered to have had complete genetic testing if all 79 exons of the dystrophin gene were examined and if this was negative, complete sequencing of the gene was performed. Diagnosis made solely by muscle biopsy or multiplex PCR analysis of a subset of exons was considered incomplete genetic testing. The national mean percentage of dystrophinopathy cases diagnosed by complete genetic testing was 63\% (SD 23). Alberta
(AB) and Newfoundland (NL) were close to the national mean, with complete genetic testing rates of $63 \%$ and $61 \%$ respectively (see Supplementary Figure 1). There was a significant difference among the provinces, with the lowest rates (mean 42, SD 6) of complete genetic testing reported in Nova Scotia (NS), Manitoba (MB), and British Columbia (BC). In contrast, the provinces of Ontario (ON), Saskatchewan (SK) and Quebec (QC) had the highest completion rates (mean 83, SD 7, t-test $\mathrm{p}=0.0018$ ). Provinces with the lowest rates of complete genetic testing (NS, 


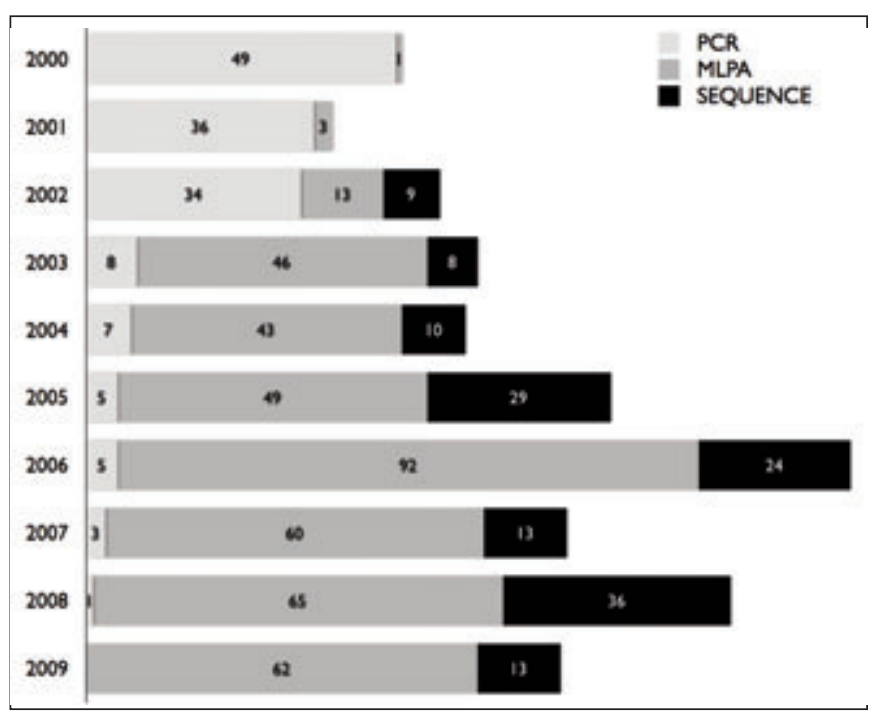

Figure 5: Change in diagnostic methods for dystrophinopathy from 2000 to 2009. PCR denotes multiplex polymerase chain reaction of subset of exons, MLPA refers to multiple ligation-probe amplification of all 79 exons, and SEQUENCE represents dystrophin gene sequencing.

MB, BC) had relied more on multiplex PCR analysis for a subset of exons $(61 \%, 59 \%$, and $47 \%)$ and less on complete gene sequencing $(17 \%, 15 \%$, and $7.2 \%$ respectively) for diagnosis. Overall, close to one-quarter (168/773 cases, 22\%) of Canadian dystro-phinopathy patients were diagnosed based on multiplex PCR analysis of the dystrophin gene without supplementary testing. Among the 200 cases with incomplete genetic testing, the phenotypes included $153 \mathrm{DMD}, 32 \mathrm{BMD}$, and 15 cases of unspecified dystrophinopathy.

\section{Discussion}

We found an estimated prevalence of dystrophinopathy of 1.5 per 10,000 males in Canada. According to Emery, the incidence of DMD was 2.9 per 10,000 , while BMD was 0.5 per $10,000^{1}$. In other reports, the incidence of DMD ranged from 1 in 7,730 (1.3 per 10,000$)$ to 1 in $3,871(2.6$ per 10,000$)$ by newborn screening in the United Kingdom ${ }^{7}$, and 1 per 4,700 (2.1 per 10,000) through a regional clinic in Nova Scotia ${ }^{8}$. A 2007 survey in the United States showed an overall prevalence of 1.3 to 1.8 per $10,000^{9}$. Results from our population-based study of the prevalence of dystrophinopathy in Canada appeared to be similar to these published reports. Consistent with two previous Canadian studies of smaller sample sizes ${ }^{10,11}$, deletions of one or more exons accounted for the majority $(n=366,64 \%)$ of the mutations. Another $63(11 \%)$ individuals had duplications and $144(25 \%)$ had point mutations. The proportion of deletions, duplications, and point mutations was similar to other recent reports on dystrophinopathy $y^{6,12,13}$.

Over the past decade, there have been considerable advances in the diagnostic approach to dystrophinopathy. Until 1988, the diagnosis usually required an open muscle biopsy, which is an invasive procedure that can be associated with bleeding, infection, and other potentially serious complications. In the

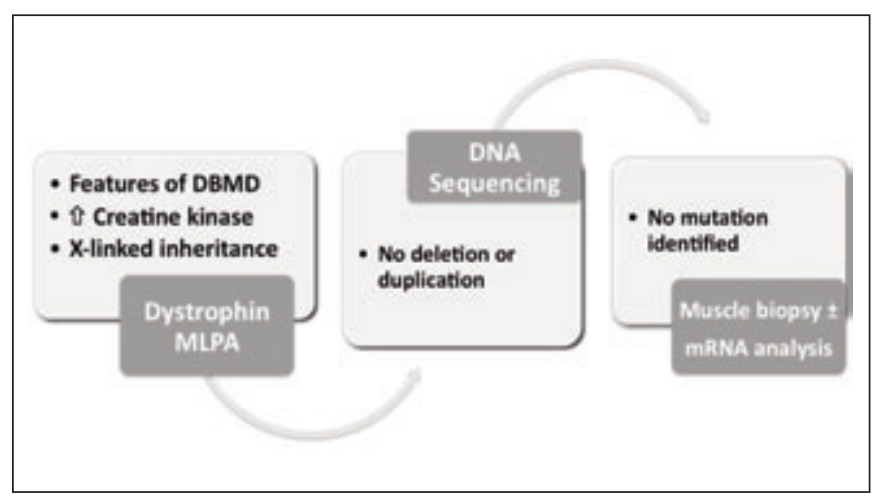

Figure 6: Recommended diagnostic approach to dystrophinopathy in Canada.

early 1990s, multiplex polymerase chain reaction provided a simple, rapid, and efficient alternative to identify common deletions occurring at one of two regions or mutational "hotspots" such as exons $2-20$ and $44-53^{14}$. Additional multiplex primer sets were added to extend the previously untested regions ${ }^{15-17}$. Although common deletions can be detected using multiplex PCR, only selective exons are examined, and precise deletion borders cannot be confirmed. In addition, duplications and small mutations will be missed. Therefore, diagnostic methods such as Southern blotting, Multiplex Amplifiable Probe Hybridization (MAPH) ${ }^{18}$, Multiplex Ligation-dependent Probe Amplification $^{19}$, other quantitative multiplex PCR assays ${ }^{11}$, whole gene sequencing, and more recently Comparative Genomic Hybridization (CGH) microarray ${ }^{20}$ are employed to detect deletions and duplications in all 79 exons.

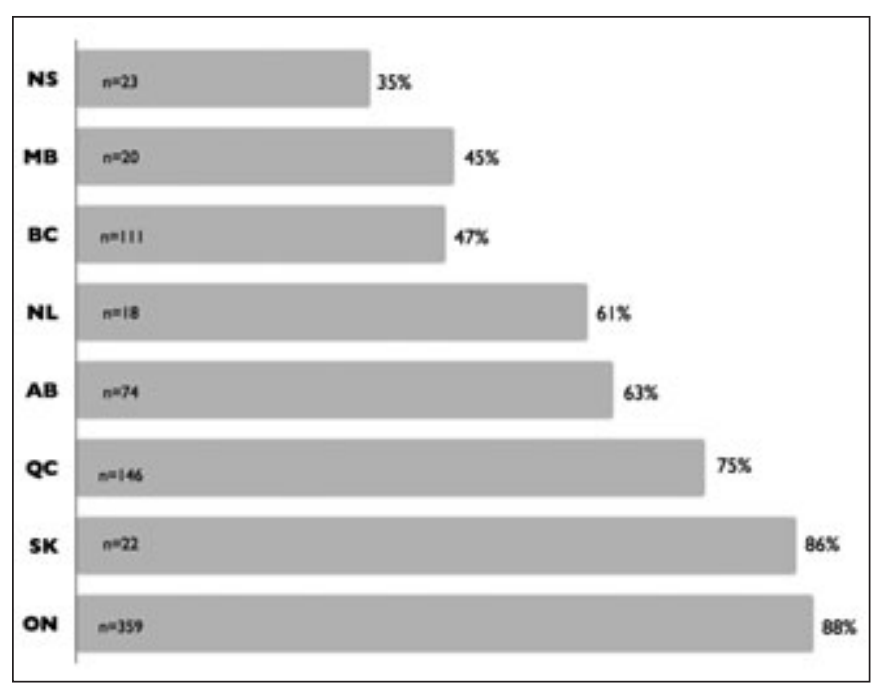

Supplementary Figure 1: Percentage of dystrophin mutation completion rates across eight Canadian provinces. NS, Nova Scotia; MB, Manitoba; $B C$, British Columbia; NL, Newfoundland; AB, Alberta; QC, Quebec; SK, Saskatchewan; ON, Ontario. 


\section{Supplementary Table 4: Confirmed duplications among} Canadian sites*

\begin{tabular}{|c|c|c|}
\hline Exons & Frequency & Phenotypes \\
\hline $1-7$ & 1 & DMD \\
\hline 2 & 13 & DMD (10), BMD (1), MD (2) \\
\hline $2-3$ & 1 & $\mathrm{MD}(1)$ \\
\hline $2-7$ & 2 & BMD \\
\hline $2-13$ & 1 & DMD \\
\hline $2-17$ & 2 & DMD (1), MD (1) \\
\hline 3 & 1 & MD \\
\hline 3-4 & 1 & BMD \\
\hline $3-5$ & 1 & DMD \\
\hline $3-7$ & 2 & DMD \\
\hline $3-9$ & 1 & DMD \\
\hline 3-11 & 1 & DMD \\
\hline $3-13$ & 1 & BMD \\
\hline 3-17 & 1 & DMD \\
\hline $4-15$ & 1 & DMD \\
\hline $5-10$ & 1 & DMD \\
\hline $5-25$ & 1 & BMD \\
\hline $8-11$ & 2 & DMD (1), MD (1) \\
\hline $8-25$ & 1 & MD \\
\hline $8-32$ & 1 & MD \\
\hline 9 & 1 & MD \\
\hline 10 & 2 & DMD \\
\hline $10-11$ & 2 & DMD \\
\hline $10-17$ & 1 & DMD \\
\hline 12 & 2 & DMD \\
\hline $12-27$ & 1 & DMD \\
\hline $13-29$ & 1 & MD \\
\hline $14-21$ & 1 & DMD \\
\hline $26-43$ & 1 & DMD \\
\hline $31-54$ & 1 & DMD \\
\hline $40-43$ & 1 & DMD \\
\hline 44 & 1 & DMD \\
\hline 45 & 1 & DMD \\
\hline $45-54$ & 1 & DMD \\
\hline $45-60$ & 1 & DMD \\
\hline $48-65$ & 1 & DMD \\
\hline $49-55$ & 1 & DMD \\
\hline 50 & 1 & DMD \\
\hline $51-52$ & 1 & DMD \\
\hline $56-62$ & 1 & DMD \\
\hline 62 & 1 & DMD \\
\hline 63-69 & 2 & DMD \\
\hline $64-67$ & 2 & DMD \\
\hline Total & 64 & \\
\hline
\end{tabular}

*Identical mutation with both Duchenne (DMD) and Becker (BMD) muscular dystrophy phenotypes is highlighted in grey; MD denotes an unspecifed phenotype.

Currently MLPA is the initial diagnostic method that is widely available across Canada for individuals with suspected dystrophinopathy. Those with negative MLPA or other quantitative PCR assays should have whole gene sequencing to identify point mutations and sequence variations in the dystrophin gene (see Figure 6) ${ }^{21}$. Approximately 1 to $2 \%$ of individuals with dystrophinopathy will not have an identifiable mutation based on gene sequencing. Muscle biopsy can then be performed to confirm the diagnosis by immunohistochemistry and/or Western blot analysis to detect the absence or alteration in the dystrophin protein. Dystrophin messenger ribonucleic acid (mRNA) can also be extracted from the muscle biopsy to determine the precise copy deoxyribonucleic acid (cDNA) sequence, including those with "identical" deletion or duplication that can occasionally produce either a DMD or a BMD phenotype due to different intronic breakpoints that affect gene splicing. Using all available diagnostic methods, it is possible to identify the dystrophin mutations and confirm the clinical phenotypes in nearly all patients with dystrophinopathy ${ }^{22,23}$.
Identification of a specific dystrophin mutation is important for accurate diagnosis, prognosis, and treatment for patients with DBMD, as well as genetic counseling for their families, which is routinely offered at all CPNG centers ${ }^{24}$. According to our study, only $74 \%$ of Canadian patients were diagnosed by currently acceptable genetic testing methods, and the same mutation completion rate (74\%) was reported by the Muscular Dystrophy Surveillance Tracking and Research Network in the United States last year ${ }^{25}$. Potential reasons for the variability in rates of diagnosis based on complete genetic testing across Canada include: a) lack of availability of dystrophin gene sequencing in most provinces; b) challenges related to obtaining funding approval to pay for out-of-province genetic testing, including dystrophin gene sequencing; c) lack of awareness regarding the potential benefits of precise mutation analysis by patients, families, or health care professionals; and d) reliance on multiplex PCR or muscle biopsy for diagnosis instead of dystrophin gene sequencing to detect point mutations by the treating physicians. This is an area that will require further investigation and is beyond the scope of our study. Other potential limitations of this study included an inability to include full clinical information due to privacy restrictions when reviewing genetic database, and the clinical phenotypes were determined by retrospective chart reviews but were not verified over time. As well, since not all CPNG members participated in this study, there may be additional patients with dystrophinopathy who were not included as they were either: a) seen elsewhere in Canada, including BMD patients followed in adult centers; b) had genetic testing through facilities in the United States or other private clinics, c) lost to follow-up, or d) died during the study period.

\section{Conclusions}

Recent scientific advances have led to potentially novel treatments for dystrophinopathy that are highly genotype specific, including: a) nonsense suppression therapy with small molecule drugs such as PTC124 or gentamicin which aims to increase ribosomal read through of premature stop codon during translation to produce a modified dystrophin $\operatorname{protein}^{26,27}$; and $\mathrm{b}$ ) exon-skipping with synthetic antisense oligonucleotide sequences to correct the reading frame shift by 'skipping over' specific exons and producing an internally truncated protein ${ }^{28,29}$. Most upcoming clinical trials for dystrophinopathy will require precise genetic and phenotypic confirmation prior to study enrollment. Dystrophin gene sequencing should therefore be readily available to all patients across Canada when MLPA testing has been uninformative. The success of novel therapeutic strategies for DBMD will ultimately depend on accurate clinical assessment and mutational analysis in potential subjects, the creation of a national or global disease-specific patient registries for comprehensive diagnosis and clinical trials readiness, and on-going interdisciplinary collaboration among academic centers to ensure that all Canadians with dystrophinopathy are eligible to receive optimal care and gain access to mutationspecific therapies. 


\section{ACKNOWLEDGEMENT}

The authors thank other CPNG members and colleagues for their support of this project: Dr. Brenda Banwell, The Hospital for Sick Children, Toronto, Ontario; Dr. Doug Biggar, Bloorview Kids Rehab, Toronto, Ontario; Dr. Peter Bridge, Alberta Children's Hospital, Calgary, Alberta; Dr. Renée-Myriam Boucher, University of Laval, Quebec City, Quebec; Dr. Gillian Hogan, Erin Oak Children's Center, Mississauga, Ontario; Dr. Noel Lowry, University of Saskatchewan, Saskatoon, Saskatchewan; Dr. Laura McAdam, Bloorview Kids Rehab, Toronto, Ontario; Dr. Hugh McMillan, Children's Hospital of Eastern Ontario, Ottawa, Ontario; Dr. David Meek, St. John's Regional Hospital, St. John's, Newfoundland; Dr. Chantal Poulin, Montreal Children's Hospital; Montreal, Quebec; Dr. Bev Prieur, Alberta Children's Hospital, Calgary, Alberta; and Dr. Jiri Vasjar, The Hospital for Sick Children, Toronto, Ontario.

\section{REFERENCES}

1. Emery AE. Population frequencies of inherited neuromuscular diseases-a world survey. Neuromuscul Disord. 1991;1(1):19-29.

2. Hoffman EP, Brown RH, Kunkel LM. Dystrophin: the protein product of the Duchenne muscular dystrophy locus. Cell. 1987; 51(6):919-28.

3. Koenig M, Monaco AP, Kunkel LM. The complete sequence of dystrophin predicts a rod-shaped cytoskeletal protein. Cell. 1988;53(2):219-28.

4. Laing NG. Molecular genetics and genetic counselling for Duchenne/Becker muscular dystrophy. In: Partridge TA, editor. Molecular and cell biology of muscular dystrophy. London: Chapman \& Hall; 1993. p. 37-84.

5. Aartsma-Rus A, Van Deutekom JC, Fokkema IF, Van Ommen GJ, Den Dunnen JT. Entries in the Leiden Duchenne muscular dystrophy mutation database: an overview of mutation types and paradoxical cases that confirm the reading-frame rule. Muscle Nerve. 2006;34(2):135-44.

6. White SJ, den Dunnen JT. Copy number variation in the genome; the human DMD gene as an example. Cytogenet Genome Res. 2006;115(3-4):240-6.

7. Bradley D, Parson E. Newborn screening for Duchenne muscular dystrophy. Semin Neonatol. 1998;3:27-34.

8. Dooley J, Gordon KE, Dodds L, MacSween J. Duchenne muscular dystrophy: a 30-year population-based incidence study. Clin Pediatr (Phila). 2010;49(2): 177-9.

9. Centers for Disease Control and Prevention (CDC). Prevalence of Duchenne/Becker muscular dystrophy among males aged 5-24 years - four states, 2007. MMWR Morb Mortal Wkly Rep. 2009; 58(40): 1119-22.

10. Gillard EF, Chamberlain JS, Murphy EG, et al. Molecular and phenotypic analysis of patients with deletions within the deletion-rich region of the Duchenne muscular dystrophy (DMD) gene. Am J Hum Genet. 1989;45(4):507-20.

11. Stockley TL, Akber S, Bulgin N, Ray PN. Strategy for comprehensive molecular testing for Duchenne and Becker muscular dystrophies. Genet Test. 2006;10(4):229-43.

12. Muntoni F, Torelli S, Ferlini A. Dystrophin and mutations: one gene, several proteins, multiple phenotypes. Lancet Neurol. 2003;2(12):731-40.

13. Yan J, Feng J, Buzin CH, et al. Three-tiered noninvasive diagnosis in $96 \%$ of patients with Duchenne muscular dystrophy (DMD). Hum Mutat. 2004;23(2):203-4.

14. Chamberlain JS, Gibbs RA, Ranier JE, Nguyen PN, Caskey CT. Deletion screening of the Duchenne muscular dystrophy locus via multiplex DNA amplification. Nucleic Acids Res. 1988;16 (23):11141-56

15. Towbin JA, Chamberlain JS, Wu DR, Pillers DA, Seltzer WK, McCabe ER. DXS28 (C7) maps centromeric to DXS68 (L1-4) and DXS67 (B24) by deletion analysis. Genomics. 1990;7(3): $442-4$
16. Beggs AH, Koenig M, Boyce FM, Kunkel LM. Detection of $98 \%$ of DMD/BMD gene deletions by polymerase chain reaction. Hum Genet. 1990;86(1):45-8.

17. Feener CA, Boyce FM, Kunkel LM. Rapid detection of CA polymorphisms in cloned DNA: application to the 5' region of the dystrophin gene. Am J Hum Genet. 1991;48(3):621-7.

18. White S, Kalf M, Liu Q, et al. Comprehensive detection of genomic duplications and deletions in the DMD gene, by use of multiplex amplifiable probe hybridization. Am J Hum Genet. 2002;71(2): 365-74.

19. Schouten JP, McElgunn CJ, Waaijer R, Zwijnenburg D, Diepvens F, Pals G. Relative quantification of 40 nucleic acid sequences by multiplex ligation-dependent probe amplification. Nucleic Acids Res. 2002;30(12):e57.

20. Hegde MR, Chin EL, Mulle JG, Okou DT, Warren ST, Zwick ME. Microarray-based mutation detection in the dystrophin gene. Hum Mutat. 2008;29(9):1091-9.

21. Bushby K, Finkel R, Birnkrant DJ, et al. Diagnosis and management of Duchenne muscular dystrophy, part 1: diagnosis, and pharmacological and psychosocial management. Lancet Neurol. 2010;9(1):77-93.

22. Baskin B, Banwell B, Khater RA, Hawkins C, Ray PN. Becker muscular dystrophy caused by an intronic mutation reducing the efficiency of the splice donor site of intron 26 of the dystrophin gene. Neuromuscul Disord. 2009;19(3):189-92.

23. Takeshima Y, Yagi M, Okizuka Y, et al. Mutation spectrum of the dystrophin gene in 442 Duchenne/Becker muscular dystrophy cases from one Japanese referral center. J Hum Genet. 2010;55 (6):379-88

24. McMillan HJ, Campbell C, Mah JK. Duchenne muscular dystrophy: Canadian paediatric neuromuscular physicians survey. Can J Neurol Sci. 2010;37(2):195-205

25. Cunniff $\mathrm{C}$, Andrews J, Meaney FJ, et al. Mutation analysis in a population-based cohort of boys with Duchenne or Becker muscular dystrophy. J Child Neurol. 2009;24(4):425-30.

26. Welch EM, Barton ER, Zhuo J, et al. PTC124 targets genetic disorders caused by nonsense mutations. Nature. 2007;447 (7140):87-91

27. Malik V, Rodino-Klapac LR, Viollet L, et al. Gentamicin-induced readthrough of stop codons in Duchenne muscular dystrophy. Ann Neurol. 2010;67(6):771-80.

28. Muntoni F, Bushby K, van Ommen G. 128th ENMC international workshop on preclinical optimization and phase I/II clinical trials using antisense oligonucleotides in Duchenne muscular dystrophy, 22-24 October 2004, Naarden, the Netherlands. Neuromuscul Disord. 2005;15(6):450-7.

29. van Deutekom JC, Janson AA, Ginjaar IB, et al. Local dystrophin restoration with antisense oligonucleotide PRO051. N Engl J Med. 2007;357(26):2677-86. 